\title{
CONSUMO DE PESCADO E OUTROS ALIMENTOS PELA POPULAÇÃO RIBEIRINHA DO LAGO GRANDE DE MONTE ALEGRE, PA - BRASIL.
}

\section{Regina Glória Pinheiro CERDEIRA', Mauro Luis RUFFINO', Victoria Judith ISAAC ${ }^{2}$}

RESUMO - O presente artigo apresenta resultados sobre o consumo de pescado e outros alimentos pela população ribeirinha do Lago Grande de Monte Alegre, no Estado do Pará, Brasil. Os dados foram coletados mensalmente, por um periodo de dois anos juntos a 35 familias de 17 comunidades, que praticam a pesca com fins comerciais e de subsistência, bem como as familias que não pescam. O consumo médio de pescado foi de $369 \mathrm{~g} /$ capita/dia, complementado com $6,1 \mathrm{~g} / \mathrm{capita} / \mathrm{dia}$ de farinha de peixe (piracui). As espécies mais consumidas foram: curimatá (Prochilodus nigricans) e acarí-bodó (Liposarcus pardalis). Em média, as famílias tiveram alguma refeiçào constituída de pescado em 6 dias de cada semana. Extrapolando para toda a população do Lago, o consumo diário de pescado é pouco mais de $3 \mathrm{t}$, chegando a $1,114 \mathrm{t} / \mathrm{ano}$.

Palavras-chave: Baixo Amazonas, consumo de pescado, lago de várzea, pesca de subsistência, piracuí.

Fish consumption and others food itens by the riverine population of the Lago Grande de Monte Alegre, PA - Brazil.

ABSTRACT - The present study shows the results on the fish consumption and other food itens by the riparian population from Monte Alegre Lake, Lower Amazon, in Pará State, Brazil, The data were colected monthly during two years jointly with 35 families from 17 fishery communities that practice the fishery with a subsistence or commercial goal, as well as families that not fish. The mean fish consumption was of $369 \mathrm{~g} /$ capita/day complemented with $6.1 \mathrm{~g} /$ capita/ day of fish flouer, called locally "piracui". The species preferred were curimata (Prochilodus nigricans) and acari-bodo (Liposarcus pardalis). Manioc flouer consumption was higher than in other regions. Families consume fish each 6 days a week. Extrapoling for all population of Lago Grande de Monte Alegre, the fish consumption is more than $3 \mathrm{t} /$ day and about 1,114t/year.

Key-words: fish consumption, floodplain lake, Lower Amazon, piracui, subsistence fishing

\section{INTRODUÇÃO}

Graças aos seus solos férteis e a alta concentração de peixes e outros vertebrados aquáticos, a várzea amazônica tem suportado algumas das maiores densidades populacionais na bacia (MEGGERS, 1971). A ocupação da várzea tem sido baseada numa estratégia de uso múltiplo envolvendo a agricultura, a pesca, o extrativismo de produtos florestais e a pecuária de pequena escala. Ao longo dos séculos, os habitantes da várzea tem variado a ênfase de suas estratégias de sobrevivência, em resposta a mudanças na economia do mercado regional, nacional e internacional (McGRATH et al., 1991).

De acordo com McGRATH et al. (1991), atualmente a base econômica da ocupação ribeirinha está seriamente ameaçada. As duas principais causas são o declínio das atividades extrativistas tradicionais e o virtual colapso da agricultura da várzea. Tais fatores estão deixando os ribeirinhos cada vez mais dependentes da pesca para obtenção de sua renda e para suprir

Projeto IARA (IBAMA/GTZ) - Av. Tapajós, 2267, Santarém, PA, 68.040-000, - Brasil

2 Museu Paraense Emilio Goeldi - Dept ${ }^{\circ}$ Zoologia, CP 399, Belém-PA, 66.040-170, Brasil 
suas necessidades básicas de subsistência (FURTADO, 1988).

O peixe é um dos recursos naturais mais abundantes e mais intensamente explorados na região amazônica. $\mathrm{O}$ número estimado de espécies biológicas existentes gira em torno de 2.500 , o que representa aproximadamente $8 \%$ dos peixes de todo o mundo, $30 \%$ dos peixes de água doce e $75 \%$ dos peixes de água doce do Brasil (COHEN, 1970; GEISLER et al., 1975).

A importância do peixe é ainda maior quando relacionado à alimentação humana. $\mathrm{O}$ consumo per capita de pescado nas cidades de Manaus e Itacoatiara foi estimado entre 100 e $200 \mathrm{~g} / \mathrm{dia}$ (SHRIMPTON \& GIUGLIANO, 1979; SMITH, 1979; AMOROSO, 1981).

O peixe é importante não somente como alimento, mas tem também grande papel na economia regional constituindose num destacado ítem das exportações, tanto na forma de pescado semiindustrializado para consumo humano, como na de peixes ornamentais. Considerando a estimativa de produção de 200.000 t/ano de BAYLEY \& PETRERE (1989) e um valor médio de primeira comercialização de aproximadamente 1US\$ por $\mathrm{kg}$ (PETRERE, 1992; ISAAC \& RUFFINO, no prelo), a pesca movimenta cerca de 200 milhões de dólares por ano. Agrega-se a isso ainda os ingressos das exportações da pesca de peixes ornamentais que oscila em torno de 1,2 milhões de dólares ao ano (LEITE \& ZUANON, 1991). Em menor escala, mas em um aumento crescente, observa-se também uma certa importância do peixe como elemento básico na fabricação de "souveniers", feitos à base de escamas de pirarucu, cabeça de piranhas, esporões de bagres, etc. (SMITH, 1979, 1981).

Talvez, o aspecto mais relevante da importância do peixe para as populações amazônicas seja a sua grande acessibilidade para as classes sociais de menor poder aquisitivo, principalmente aquelas que habitam as regiões de várzea onde, praticamente, inexiste uma pecuária efetiva e é deficiente o sistema de abastecimento através dos grandes centros comerciais. De acordo com SANTOS et al. (1991), a maior constatação da importância do pescado para estas populações provém do fato de que a maioria dos povoados/comunidades da região situam-se ao longo dos rios e lagos, portanto, com acesso direto ao peixe.

Apesar da importância da pesca na Amazônia, como fonte de proteína e de renda, existe uma falta de reconhecimento por parte das autoridades competentes. Prova disso é a inexistência de séries históricas de estatísticas sobre a produção total de pescado.

A pesca no interior da Amazônia pode ser dividida em uma atividade de subsistência, realizada por um ou dois pescadores a bordo de uma canoa ou bote e com poucas e relativamente simples artes de pesca, ou uma atividade comercial, na qual os canoeiros vendem parte de sua produção para embarcações que possuem caixas ou urnas com gelo, chamadas de "geleiras" e que transportam o pescado para os centros urbanos para a sua comercialização nos mercados das cidades da região, ou até mesmo para sua exportação para outros estados da União e para o exterior, através dos frigoríficos 
(ISAAC \& BARTHEM, 1996).

Visando conhecer a produção pesqueira no Baixo Amazonas, o Projeto IARA (IBAMA, 1995) implementou um sistema de coleta de dados estatísticos de desembarque pesqueiro em vários municípios da região (ISAAC \& RUFFINO, no prelo). No entanto, a produção gerada pela pesca de subsistência normalmente é excluída das estatísticas oficiais de desembarque pesqueiro, pois frequentemente destina-se ao consumo das próprias familias dos pescadores ou ao comércio estritamente local.

Segundo BAYLEY \& PETRERE (1989), a produção pesqueira gerada pela pesca de subsistência pode ser estimada com maior precisão se estudado o consumo médio per capita de pescado (por espécie) regionalmente e multiplicando este valor pelo número de habitantes. Estimar captura/pescador/dia na pesca de subsistência, implica no conhecimento do número total de pescadores para poder realizar extrapolações.

O presente trabalho apresenta os resultados sobre o consumo médio per capita de pescado, utilizado como um estimador da produção pesqueira gerada pelas comunidades ribeirinhas. $\mathrm{O}$ estudo não avalia qualitativamente os ítens alimentares da população, mas cita a composição quantitativa dos principais alimentos das famílias da região, enfatizando $o$ alto consumo de peixe $\mathrm{e}$ farinha de mandioca, que são as principais fontes de proteína, $\mathrm{O}$ estudo foi desenvolvido no Lago grande de Monte Alegre, um lago de várzea de aproximadamente 50.000 ha localizado à margem direita do rio Amazonas, no município de Monte Alegre, PA.

\section{MATERIAL E MÉTODOS}

Os dados para o presente estudo foram coletados de maio de 1993 a março de 1995 , após a realização de um censo comunitário (ISAAC et al., no prelo). As famílias foram divididas em 3 estratos, de acordo com o tipo de pesca que praticam: PS para as familias que praticam a pesca para a sua própria subsistência; PC para as famílias que exercem a pesca como atividade comercial e NP para as famílias que não pescam.

De cada estrato, foram sorteadas aproximadamente $2 \%$ do total de famílias, resultando na escolha de 11 familias da pesca comercial, 19 da pesca de subsistência e 5 famílias que não pescam, totalizando 35 famílias distribuidas em 17 comunidades do Lago Grande de Monte Alegre-PA. Pelo menos duas pessoas de cada familia foram treinadas por um grupo de pesquisadores do Projeto IARA para manipular as balanças de $3 \mathrm{~kg}$ (precisão 50 g) e preencherem formulários para a pesquisa. Os dados foram colhidos durante uma semana de cada mês. Seleção aleatória simples (COCHRAN, 1977) foi o critério usado para decidir as semanas para a amostragem. Foram colhidas as seguintes informações: tipo de alimento consumido pela familia, quantidade $(\mathrm{em} \mathrm{g}$ ), período do dia consumido e número de pessoas por familia (ANEXO 1). No caso de peixes, os mesmos foram separados por espécie e pesados inteiros, sem tratamento prévio. Os ovos foram considerados por unidade, e o leite medido $\mathrm{em} \mathrm{ml}$. As espécies de peixes foram referidas com seus nomes vulgares, mesmo sabendo que existem muitas categorias taxonômicas para cada nome 
vulgar (ANEXO 2), pois era a única classificação viável e unívoca.

A coleta de dados foi monitorada através de visitas periódicas às famílias, oportunidade em que os formulários preenchidos foram recolhidos e novos formulários foram fornecidos. O total de formulários preenchidos foi de 3.918 , o que representa aproximadamente, $70 \%$ do total previsto.

Os dados foram digitados em um banco de dados previamente elaborado, utilizando o programa DataEase (versão 4.01).

A análise dos dados constou da obtenção de valores médios da quantidade de peixe e outros alimentos consumidos por família, por estrato, por dia. O consumo diário de cada alimento no grupo familiar foi dividido pelo número de pessoas que participaram das refeições. Os dias em que um determinado alimento não foi consumido, foram computados com valor zero. A média de consumo de cada alimento per capita foi obtido para toda a população amostrada, por tipo de pesca e por mês. A média de consumo/capita/ anual foi obtida multiplicando a média diária por 365 dias. $O$ consumo anual de peixe para toda a população do Lago Grande de Monte Alegre foi estimado multiplicando-se o consumo anual per capita pelo número de moradores do lago, por estrato de pesca. A frequência média de consumo de cada alimento foi estimada a partir do número de vezes que houve consumo na semana dividido pelo número de dias da semana.

Para avaliar a existência de diferenças significativas entre as famílias em relação ao consumo de pescado, animais de criação doméstica e animais de caça, aplicou-se um teste "t" entre as médias gerais por estrato, para nível de significância de 99\% (COCHRAN, 1977), uma vez que as mesmas apresentam distribuição normal.

\section{RESULTADOS}

O tamanho médio das famílias amostradas foi de 7 pessoas, com um número mínimo de 1 e máximo de 21 pessoas por família. A frequência de consumo de peixe foi de 0,81 , ou seja, de cada 10 dias, em 8 dias houve pelo menos uma refeição constituída de pescado, ou 6 dias por semana. Considerando essa frequência ao longo de 365 dias, tem-se que os ribeirinhos consomem peixe em 296 dias do ano.

O consumo médio diário per capita de peixe (em peso bruto) foi de $369 \mathrm{~g}$ e o anual de $135 \mathrm{~kg}$, sendo que, em média, cada família consome, por ano, cerca de $943 \mathrm{~kg}$ de peixe. Observa-se que o consumo médio diário eleva-se a partir de agosto de 1993, no período da seca, enquanto que em 1994 esse aumento ocorre apenas no mês de setembro (Fig 1).

Analisando o consumo médio diário entre as familias dos três estratos de pesca, tal padrão se repete, porém evidencia-se um menor consumo de peixe das familias que não exercem a atividade pesqueira (média de $293 \mathrm{~g} /$ dia) quando comparado àquelas que pescam para subsistência (média de $381 \mathrm{~g} / \mathrm{dia}$ ) e comercialmente (média de 383 g/dia) (Tab 1; Fig 2).

Supondo uma quebra de $40 \%$ do peso do peixe inteiro, relativo às vísceras, ossos e escamas (SMITH, 1979), tem-se que o consumo médio per capita/dia de 369 g cai para 221,4 g per capita/dia. 


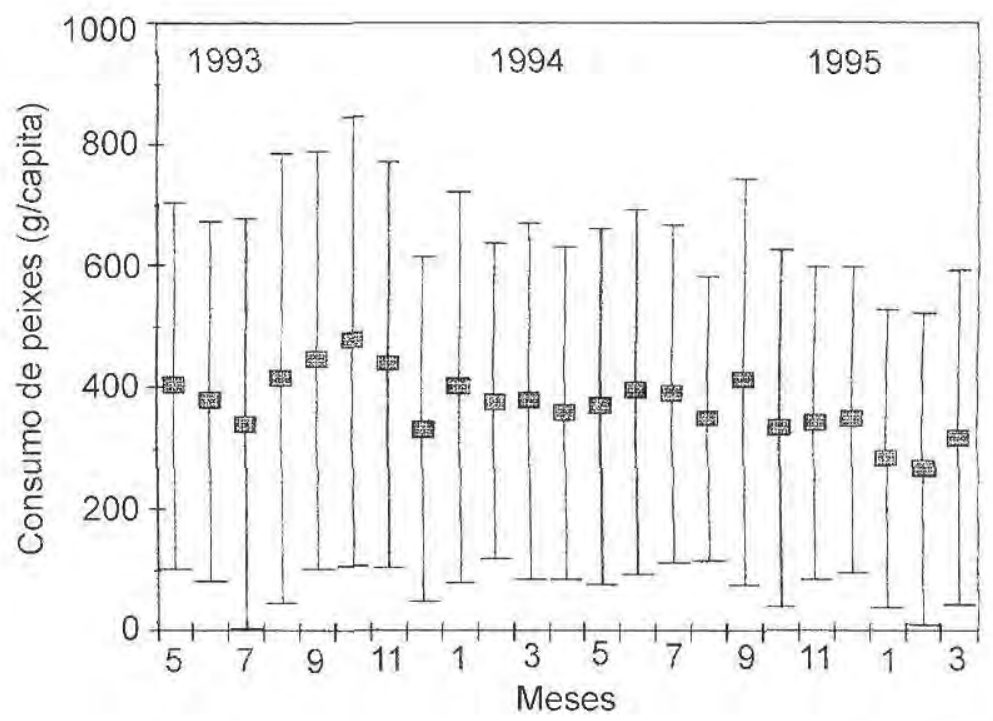

Figura 1. Variação mensal do consumo médio/capita/dia de peixe e o desvio padrão de 35 famílias do Lago Grande de Monte Alegre, no periodo de maio de 1993 a março de 1995.

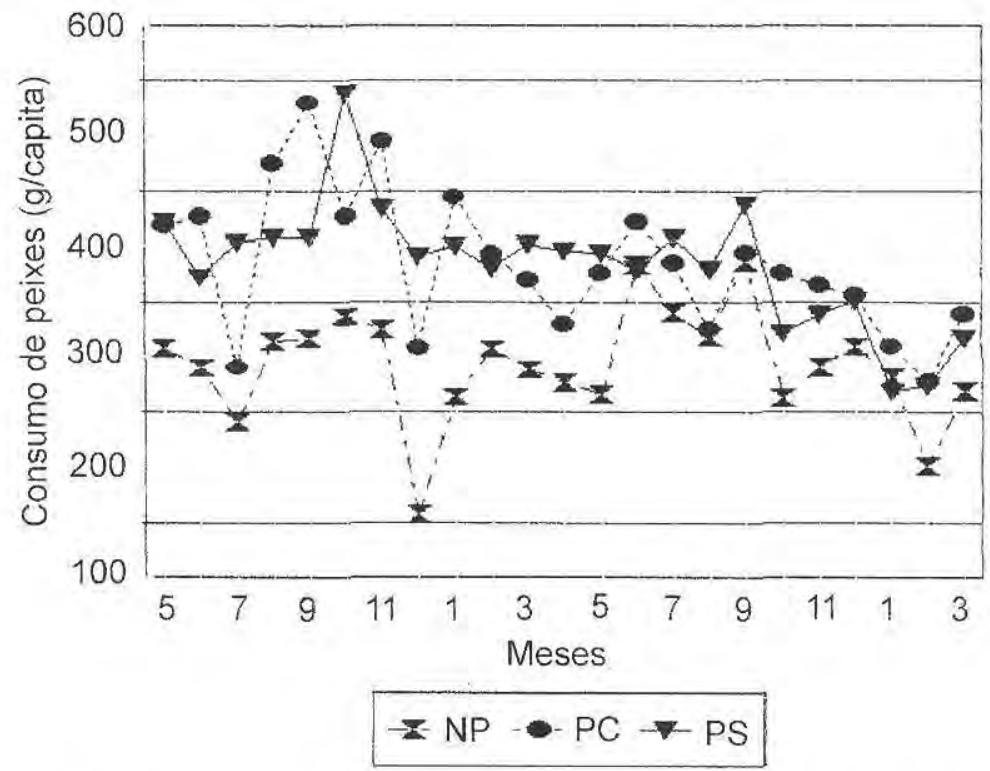

Figura 2. Variação mensal do consumo de pescado para os diferentes tipos de familias do Lago Grande de Monte Alegre, no período de maio de 1993 a março de 1995. (NP= Não pesca; PS= Pesca de subsistência e PC= Pesca comercial). 
Considerando o número total de moradores do Lago Grande de Monte Alegre e o consumo médio per capita por dia, por estrato, chega-se a um valor $3.053 \mathrm{~kg} / \mathrm{dia}$, a partir do qual, extrapolando para todo o ano, obtemse 1.114 t/ano (Tab. 1).

Apesar de não haver diferenças significativas $(P>0,01)$ entre $o$ consumo médio per capita de peixe das familias que pescam para subsistência (PS) e para a pesca comercial (PC), observa-se diferenças significativas $(P>0,01)$ entre as famílias que não pescam e aquelas que pescam (PS e PC juntas), cujas médias estimaram-se em $293 \mathrm{~g} /$ capita/dia e 382 g/capita/dia (média entre PS e PC), respectivamente (Tab. 1). o acarí (Liposarcus pardalis), representando, cada um, cerca de $10 \%$ do consumo total. A pescada (Plagioscion spp.) e o surubim (Pseudoplatystoma spp.) contabilizam cada um 7\% do total consumido (Fig. 3).

Além do pescado, os ribeirinhos do Lago Grande de Monte Alegre consomem pequenos animais criados em suas propriedades como frango, porco ou pato. Provindo da criação em pequena escala, há também consumo de carne bovina. Esporadicamente, fazem parte também da dieta animais obtidos através da caça, como marreca (Anas spp., Amazonetta spp.), pato do mato (Mergus spp.), maguari (Ciconia maguari), passarão (Mycteria americana), saracura (Rallus spp., Aramides spp.), tracajá

Tabela 1. Consumo médio/capita e total por estrato de pesca para o Lago Grande de Monte Alegre, no período de abril de 1993 a março de $1995 \mathrm{NP}=$ não pesca; $\mathrm{PS}=$ pesca de subsistêneia e $\mathrm{PC}=$ pesca comercial.

\begin{tabular}{ccccc}
\hline $\begin{array}{c}\text { Tipo de } \\
\text { pesca }\end{array}$ & $\begin{array}{c}\text { № de } \\
\text { moradores }\end{array}$ & $\begin{array}{c}\text { Consumo } \\
\text { médio/capita/dia } \\
(\mathrm{g})\end{array}$ & $\begin{array}{c}\text { Consumo } \\
\text { médio/dia/estrato } \\
(\mathrm{kg})\end{array}$ & $\begin{array}{c}\text { Consumo } \\
\text { médio/ano/estrato } \\
(\mathrm{t})\end{array}$ \\
\hline NP & 1.275 & 293 & 374 & 136 \\
\hline PS & 4.783 & 381 & 1.822 & 665 \\
\hline PC & 2.217 & 383 & 849 & 309 \\
\hline Média/Total & 8.275 & 369 & 3.053 & 1.114 \\
\hline
\end{tabular}

Outra forma de consumo de pescado entre as familias do Lago Grande de Monte Alegre é a farinha de peixe, conhecida na região como "piracuí" que geralmente é elaborada a partir da carne do acari-bodó (Liposarcus pardalis). $\mathrm{O}$ consumo médio per capita/dia de piracuí é $6,1 \mathrm{~g}$ (Tab. 2), o qual contribui para aumentar, levemente, 0 consumo real de peixe de $221,4 \mathrm{~g}$ para $227,1 \mathrm{~g} /$ capita/dia.

Dentre as espécies mais consumidas estão o curimatá (Prochilodus nigricans) $\mathrm{e}$
(Podocnemis spp.), paca (Agouti spp.), tatu (Priodontes spp.), e jacaré (Melanosuchus spp., Paleosuchus spp.).

O consumo médio de animais de criação doméstica e animais de caça é $105,36 \mathrm{~g} /$ capita/dia e 4,93 g/capita/dia, respectivamente. A frequência de consumo de animais de criação doméstica é de 0,38 , ou seja, 4 de cada 10 dias, apresentaram animais provindos da criação doméstica no cardápio. Dois em cada 100 dias, as famílias têm 


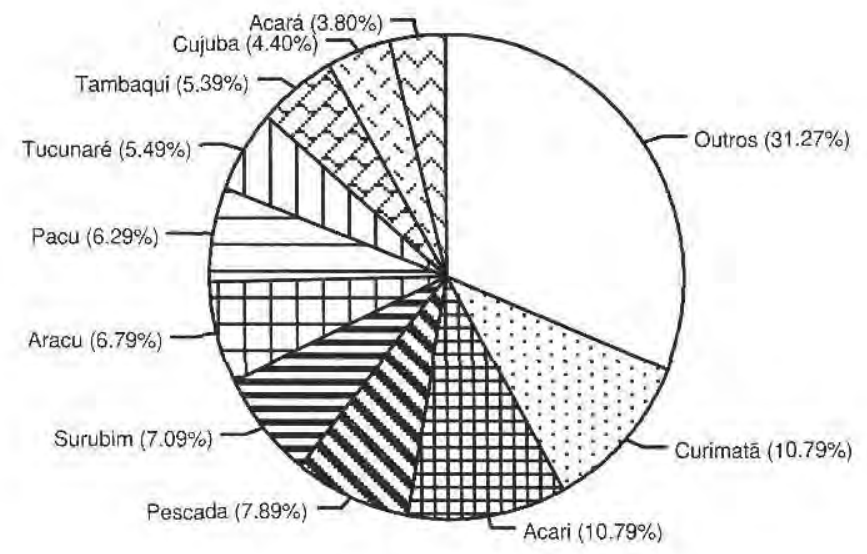

Figura 3. Proporção das espécies de peixes mais consumidas por 35 familias do Lago Grande de Monte Alegre, no período de maio de 1993 a março de 1995.

refeições preparadas com animais de caça, com uma frequência de 0,02 . O consumo médio de animais de criação doméstica variou bastante, contudo é mais elevado durante o fim e o início do ano, quando começam as chuvas (Fig.4). Já o consumo de animais de caça apresentou-se mais acentuado nos meses da estação seca entre outubro e novembro (Fig. 5). Não houve diferenças significativas $(\mathrm{P}>0,01)$ no consumo de animais de caça e animais de criação doméstica entre as familias dos vários estratos de pesca. O consumo de carne de gado é de $4,3 \mathrm{~g} /$ capita/dia e a frequência de 0,22 ( 2 de cada 10 dias) (Tab. 2 )

Entre outros tipos de alimentos, a farinha e o leite foram os mais consumidos com aproximadamente $231 \mathrm{~g} /$ capita/dia e $104 \mathrm{ml} /$ capita/dia, respectivamente (Tab. 2).

Não foi observado o consumo de hortaliças, com exceção de algumas familias de uma única comunidade.

\section{DISCUSSÃO}

Tradicionalmente, o peixe é o componente mais importante para a alimentação das famílias ribeirinhas e urbanas da região. Estudos anteriores como o de HONDA et al. (1975) mostram um grande consumo de pescado na cidade de Manaus-AM.

A Tabela 3 apresenta um resumo das estimativas de consumo de peixes obtidas por vários autores para a região amazônica. Nota-se que o valor estimado no presente estudo é mais elevado que os estimados por outros autores. Contudo, recentemente, 


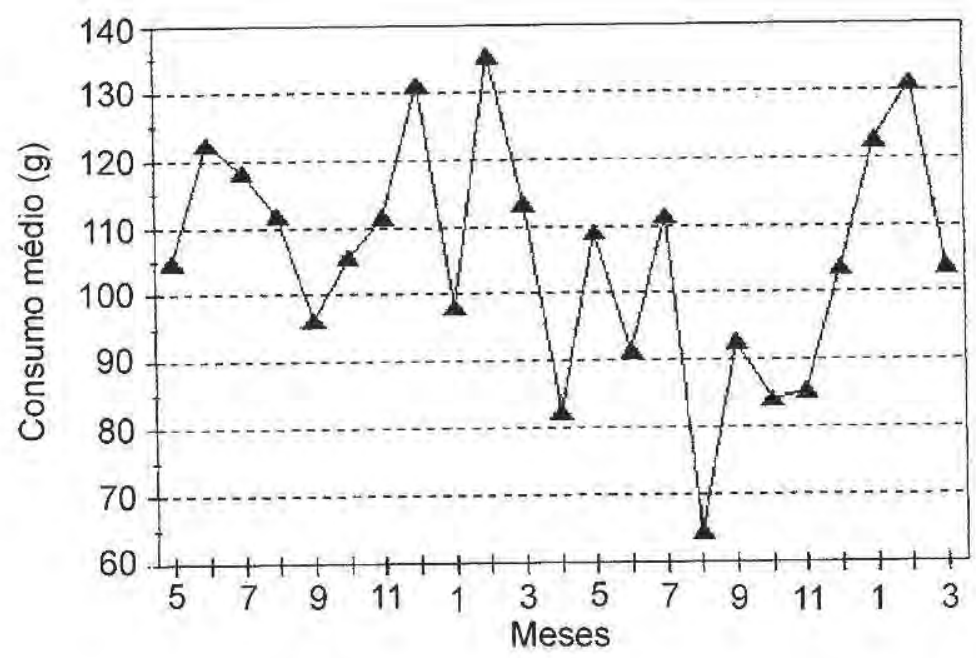

Figura 4. Variação mensal do consumo médio/capita/dia de animais de criação doméstica de 35 familias do Lago Grande de Monte Alegre, no período de maio de 1993 a março de 1995.

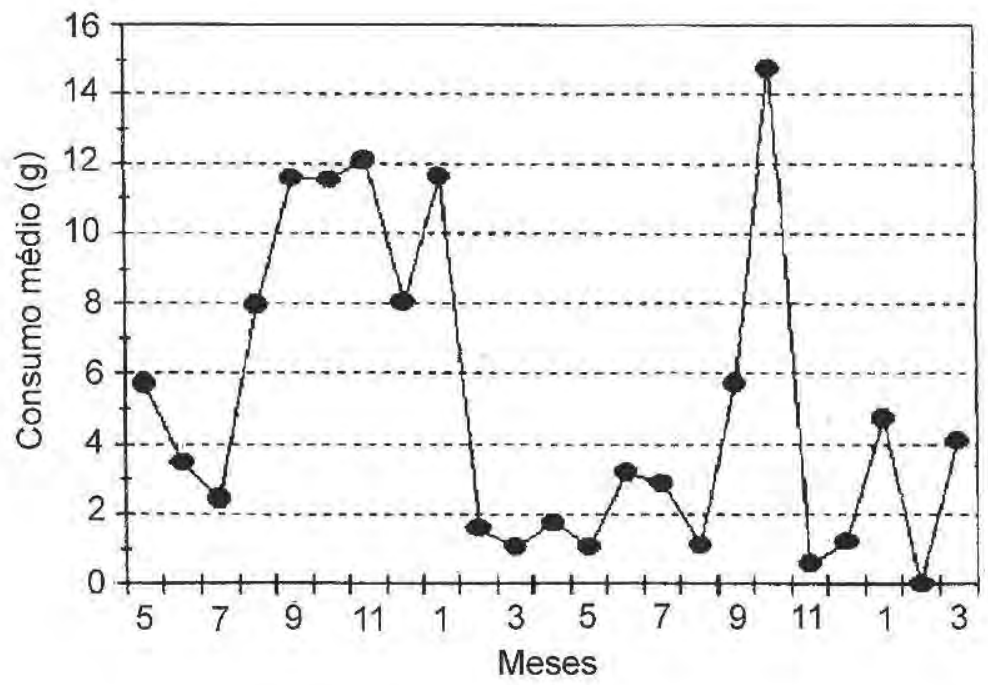

Figura 5. Variação mensal do consumo médio/capita/dia de animais de caça de 35 familias do Lago Grande de Monte Alegre, no período de maio de 1993 a março de 1995. 
Tabela 2. Itens alimentares mais frequentes e mais consumidos por 35 familias do Lago Grande de Monte Alegre, no período de maio de 1993 a março de 1995.

\begin{tabular}{lccc}
\hline Tipo de alimento & $\begin{array}{c}\text { Consumo/capital/dia } \\
(\mathrm{g})\end{array}$ & $\begin{array}{c}\text { Consumo/capital/ano } \\
(\mathrm{kg})\end{array}$ & $\begin{array}{c}\text { Frequência média de } \\
\text { consumo }\end{array}$ \\
\hline Pescado & 369,00 & 135,00 & 0,81 \\
\hline Farinha & 231,00 & 84,50 & 0,94 \\
\hline Arroz & 48,00 & 17,52 & 0,50 \\
\hline Tapioca & 26,00 & 9,38 & 0,34 \\
\hline Feijão & 10,00 & 3,60 & 0,11 \\
\hline Batata & 7,00 & 2,60 & 0,05 \\
\hline Piracui & 6,10 & 2,22 & 0,09 \\
\hline Carne de gado & 4,33 & 1,58 & 0,22 \\
\hline Macacheira & 0,40 & 0,13 & 0,001 \\
\hline Macarão & 0,40 & 4,00 & 0,16 \\
\hline Conserva & 0,25 & 0,10 & 0,003 \\
\hline Charque & 0,05 & 0,01 & 0,002 \\
\hline Ovos de quelônio & 0,05 & 0,01 & 0,02 \\
\hline Queijo & 0,03 & 0,01 & 0,0005 \\
\hline Leite & $104 \mathrm{ml}$ & 381 litros & 0,53 \\
\hline Ovos de galinha & 0,3 unid. & 0,10 unid. & 0,26 \\
\hline
\end{tabular}

BATISTA \& FREITAS (1995) estimaram $550 \mathrm{~g} /$ capita/dia na Amazônca Central e MITLEWSKI et al. (no prelo) obtiveram $600 \mathrm{~g} /$ capita/dia para o Lago Jauarí/Botos no Baixo Amazonas. Porém, tais levantamentos foram obtidos somente através de entrevistas, sem pesar o alimento. $\mathrm{O}$ consumo per capita anual estimado no presente estudo foi de $135 \mathrm{~kg}$ que, considerando a quebra de $40 \%$, referente às partes não comestiveis do peixe, este valor cai para $81 \mathrm{~kg}$. Esta estimativa supera os valores de $55 \mathrm{~kg} /$ capita/ano estimado por SHRIMPTON \& GIUGLIANO (1979) para a cidade de Manaus, $38 \mathrm{~kg}$ estimados por SMITH (1979) para Itacoatiara e $72 \mathrm{~kg} /$ capita/ ano mencionados por VIEIRA \& HARTMANN (1989) também para as familias do Lago Grande de Monte Alegre (Tab 3.).

A importância do peixe na dieta do ribeirinho é, ainda, mais significativa pelo seu valor nutritivo, contribuindo para uma alimentação mais sadia e equilibrada. De acordo com SMITH (1979), o percentual de gordura e proteína das principais espécies consumidas varia de 2 a $24,8 \%$ e de 13,8 a $18 \%$, respectivamente. Considerando que um adulto necessita cerca de $0,6 \mathrm{~g}$ de proteína animal por $\mathrm{kg}$ de peso corpóreo/dia (SMITH, 1979). O consumo real per capita de $227 \mathrm{~g}$ de peixe estimado no presente trabalho seria mais do que suficiente para cobrir a necessidade proteica de uma pessoa de aproximadamente $60 \mathrm{~kg}$.

O consumo de animais de criação doméstica variou ao longo do período de estudo, não apresentando nenhum padrão, já o consumo de animais de caça diminui no período das chuvas, pois nesta época a caça torna-se mais dificil em função da eliminação dos rastros e vestígios dos animais provocada pelas chuvas. Segundo os prórpios 
Tabela 3. Consumo diário per capita de pescado, em algumas localidades da região amazônica, de acordo com diferentes autores.

\begin{tabular}{ccc}
\hline Consumo $(\mathrm{g})$ & Região & Fonte \\
\hline 86,71 & Manaus & HONHAet. al.(1975) \\
\hline 104,00 & Manaus & AMOROSO (1981) \\
\hline 121,70 & Manaus & GIUGLIANOet. al.(1978) \\
\hline 150,60 & Manaus & SHRIMPTON \& GIUGLIANO (1978) \\
\hline 194,00 & Itacoatiara & SMITH (1979) \\
\hline 369,00 & Baixo Amazonas & Presente estudo \\
\hline
\end{tabular}

ribeirinhos, o consumo destes animais é esporádico e complementa a dieta de peixe. A criação doméstica de animaís é um hábito dos moradores do Lago Grande de Monte Alegre, servindo de suporte alimentício ou ainda como objeto de troca e/ou venda em viagens ocasionais à cidade (ISAAC et al., no prelo). O consumo de carne de gado é esporádico, porém o consumo de leite é frequente. O consumo de carne de gado é bem inferior ao encontrado por SHRIMPTON \& GIUGLIANO (1979), provavelmente porque, na região, o gado é utilizado como forma de capitalização, onde o animal representa um valor estável que pode servir, por um lado, como reserva econômica em casos de emergência ou até mesmo como garantia para empréstimos, além de servir como meio de transporte (ISAAC et al., no prelo).

Por terem acesso ao peixe mais frequentemente, logicamente, as familias que pescam, tanto as da pesca comercial como as de subsistência, apresentam um consumo médio per capita um pouco maior que o consumo das famílias que não pescam, as quais adquirem o peixe através de compra ou doação. As famílias que não pescam geralmente se ocupam quase que integralmente de outras atividades de extrativismo e agricultura familiar (ISAAC et al., no prelo).

A farinha de mesa, o principal subproduto da raiz de mandioca, envolve cerca de $90 \%$ da produção regional da cultura e sua fabricação é em regime familiar (ALBUQUERQUE \& CARDOSO, 1983). A composição de nutrientes digestíveis totais da raiz da mandioca é semelhante às dos seus subprodutos, apresentando aminoácidos e carotenos (CAMARÃO et al., 1993). A farinha consumida na região é a farinha amarela, a qual é fonte de vitamina A e possui alto teor de energia, além de minerais como o ferro (SHRIMPTON \& GIUGLIANO, 1979, AMOROSO, 1981). Seu consumo foi superior ao estimado por SHRIMPTON \& GIUGLIANO (1979) de $122,5 \mathrm{~g}$ de farinha d'água e 19,8 $\mathrm{g}$ de farinha seca, atingindo $231 \mathrm{~g} /$ capita/dia.

Quanto ao consumo de leite fresco, SHRIMPTON \& GIUGLIANO (1979) estimaram em 0,9 ml, Já BATISTA et al. (submetido), baseado em levantamentos realizados por entrevistas, encontraram valores entre 220 e $310 \mathrm{ml}$, quase o dobro encontrado no presente estudo (104 ml/capita/dia).

O consumo de ovos de 0,45 a 0,91 unidades/capita/dia, estimado por 
BATISTA et al. (submetido), apesar de ligeiramente maior, aproxima-se do valor consumido entre as famílias do Lago Grande de Monte Alegre que é de 0,3 ovos/capita/dia.

Dentre as espécies de peixes preferidas pela população ribeirinha, a maior parte delas é constituida por peixes de escama, tais como curimatã, pescada, aracu, pacu, tucunaré, tambaqui, acará entre outros. Apenas o surubim, acari e cujuba foram consumidos entre os Siluriformes, também conhecidos como peixes lisos. Outras espécies de Siluriformes como a dourada (Brachyplatystoma flavicans), piramutaba (Brachyplatystoma vaillantii) ou mapará (Hypophthalmus spp.) são destinados principalmente a comercialização nos grandes centros urbanos apesar de terem sido consumidos em menor quantidade, corroborando com os achados de ISAAC et al. (1996). Em geral, os Siluriformes, particularmente dourada, piramutaba ou mapará, são peixes rejeitados pela população ribeirinha devido ao seu alto teor de gordura e sua carne é considerada "reimosa", As pessoas supõem que essa carne causa Hanseníase e outras doenças (JUNK, 1984). BEGOSSI \& BRAGA (1992), trabalhando com hábitos alimentares das populações ribeirinhas do rio Tocantins, concluem que as preferências alimentares podem ser parcialmente explicadas pelo hábito alimentar dos peixes e por sua utilidade, principalmente o uso medicianal. De acordo com ROSS (1978) esses tabus também tem sido interpretados como uma maneira de minimizar a sobrexploração de certas espécies.

Considerando o volume de pescado capturado no Lago Grande de Monte Alegre durante 1 ầo (setembro/93 a agosto/94), desembarcado nos municípios de Abaetetuba, Belém, Breves, Macapá, Monte Alegre e Santarém de 1.094 t (RUFFINO, no prelo) e o consumo anual de pescado para toda a população do lago de 1.114 t estimado no presente trabalho, chega-se a $2.208 \mathrm{t}$ de pescado que são extraídos anualmente do Lago Grande de Monte Alegre.

ISAAC et al. (no prelo), baseados em dados de captura e esforço da comunidade de Ilha do Flexal e correlações de WELCOMME (1985), estimaram a produção potencial do Lago Grande de Monte Alegre entre $1.500 \mathrm{t}$ a 2.900 t/ano. Considerando a área do Lago Grande de Monte Alegre de 57.611 ha (VIEIRA \& HARTMANN, 1989) e os valores de biomassa de 10,4 a 47,3 kg/ha estimadas por RUFFINO $(1992,1994)$ para o mesmo lago, o potencial pesqueiro, em termos de biomassa de peixe, para todo o lago seria de 599 t a 2.725 t, incluidos todos os tipos de peixes, inclusive aqueles que não são consumidos pela população.

Porém, a partir de entrevistas aos pescadores, a captura anual no Lago Grande de Monte Alegre foi estimada em mais de 8.000 t por CERDEIRA et al. (no prelo).

De todas as maneiras, os resultados deste trabalho salientam a importância do peixe na alimentação do ribeirinho, indicando que uma importante parte da produção pesqueira é destinada à alimentação.

\section{AGRADECIMENTOS}

Os autores agradecem ao IBAMA, GOPA-GTZ e ao PTU/CNPq pelo aporte financeiro para a coleta dos dados e, especialmente, à Biol. Vera Lúcia 
Cardoso Rocha, Biol. Adriane Hager e ao Téc. Agr. Gerson Luiz Rêgo pelo auxílio nos trabalhos de campo. Especial agradecimento a todas as familias e pessoas do Lago Grande de Monte Alegre que colaboraram para que este trabalho fosse possivel.

\section{Bibliografia Citada}

AMOROSO, M.C.M, 1981. Alimentação em um bairro pobre de Manaus. Acta Amazonica (Supl.), 11(3): 43 p.

ALBUQUERQUE, M. DE .; CARDOSO, E.M.R. 1983. Utilização da mandioca na Amazônia. EMBRAPA; CPATU. Belém-PA. p 11.

BATISTA, V.S.; FREITAS, C.E.C., BRASIL, D.F.1995. Characterization of the actual status of commercial and communitary fisheries in the Central Amazonian Region. $X X Y I$ Congress of International Association of Theorical an Applied Limnology. Abstract. p. 68 .

... ; SILVA, A.J.I.; FREITAS, C.E. DE C.; BRASIL, D.F. (submetido). Caracterization of the fishery in riverine communities in the Low-Solimões/High-Amazon region. p. 21.

BAYLEY, P.B.; PETRERE Jr, M. 1989. Amazon fisheries: assessment methods, current status, and management options. p. 385-398. In: DODGE, D.P. (ed.). Proceedings of the International Large River Symposium. Can. Spec. Publ. Fish. Aquat. Sci., 106.

CERDEIRA, R.G.P.; ISAAC, V.J.; RUFFINO, M.L. (no prelo). Captura de pescado pelas comunidades ribeirinhas do Lago Grande de Monte Alegre, PA - Brasil. IBAMA. Coleção Meio Ambiente. Série Estudos de Pesca.

BEGOSSI, A. ; BRAGA, F.M. DE S. 1992. Food taboos and folk medicine among fishermen from the Tocantins River (Brazil). Amazoniana. XII (1): 101 - 118 .

COHEN, D.M. 1970. How many recent fishes are there? Proc. Calif. Acad. Sciences, Ser. 4, 38:341-346.

COCHRAN, W.G. 1977. Sampling techniques. Publ. John Wiley; Sons, Inc. 428 p.

CAMARÃO, A.P., BATISTA, H.A.M., LOURENÇO JUNIOR, J. DE B.,
CARDOSO, E.M.R. 1993. Utilização da mandioca na alimentação de ruminantes na Amazônia. EMBRAPA.; CPATU. 40 p.

FURTADO, L. G. 1988. Os caboclos pescadores do baixo rio Amazonas e o processo de mudança social e econômica. Ciências Sociais e o Mar no Brasil. II Programa de Pesquisa e Conservação de Areas Umidas no Brasil. São Paulo.

GEISLER, R.; KNÖPPEL, H.A, ; SIOLI, H. 1975. The ecology of freshwater fishes in Amazonia, present status and future tasks for research. Appl. Sci. Develop., 2:144-162.

GIUGLIANO, R., SHRIMPTON, R., ARKCOLL, D.B., GIUGLIANO, L.G., PETRERE M. 1978. Diagnóstico da realidade alimentar e nutricional do Estado do Amazonas. Acta Amazonica, 2 (2): 54 p.

HONDA, E.M.S., CORREA, C.M., CASTELO, F.P., ZAPELINI, E.A. 1975.Aspectos gerais do pescado no Amazonas. Acta Amazonica, 5 (1): 87-94.

IBAMA. 1995. PROJETO IARA - Administração dos recursos pesqueiros na região do Médio Amazonas (Pará e Amazonas). IBAMA. Coleção Meio Ambiente. Série Estudos de Pesca, (15): $100 \mathrm{p}$.

ISAAC, V.J.; BARTHEM, R.B. 1996. A pesca na Amazônia brasileira. Bol. Mus. Para. Emilio Goeldi, sér. Antropol.

.... ; MITLEWSKI, B; RUFFINO, M.L.; OLIVEIRA, P.R.S. (no prelo). Lago Grande de Monte Alegre: Uma análise censitária de suas comunidades pesqueiras. IBAMA. Coleção Meío Ambiente. Série Estudos de Pesca.

.... ; MILSTEIN, A. ; RUFFINO, M.L. 1996. A pesca artesanal no Baixo Amazonas Análise multivariada da captura por espécie. Acta Amazonica, 26(3):185-208.

.... ; RUFFINO, M.L. (no prelo). A estatística pesqueira no Baixo Amazonas: Experiência do Projeto IARA. IBAMA. Coleção Meio Ambiente. Série Estudos de Pesca.

JUNK, W.J. 1984. Ecology of the "várzea". Floodplain of Amazonian white-waters rivers. In: The Amazon Liminology and Landscape ecology of a mighty tropical 
river and its basin. SIOLI, H. (Ed.). Dr. Junk Publishers. Dordrecht. p. 215-243.

LEITE, R.G. ; ZUANOM, J.A.S. 1991. Peixes ornamentaís - Aspectos de comercialização, ecologia, legislação e propostas de ações para um melhor aproveitamento. pp.327-331. In: VAL,A.L.; FIGLIUOLO, R.; FELDBERG, E. (eds.). Base Cientificas para estratégias de preservação e desenvolvimento da Amazônia: Fatos e perspectivas. INPA, Manaus, $440 \mathrm{p}$.

McGRATH, D.G.; CALABRIA, J.; AMARAL, B.; FUTEMA, C. ; CASTRO, F, 1991. Varzeiros, geleiros e o manejo dos recursos naturais na várzea do Baixo Amazonas. Cadernos do NAEA, (11);91-125.

MEGGERS, B. 1971. Amazonia: Man and Culture in a Counterfeit Paradise. Chicago, Aldine.

MITLEWSKI, B; OLIVEIRA, P.R.S.; RUFFINO, M.L.; CASTRO, F (no prelo). Lago Jauari/ dos Botos. Resultados do censo estatístico comunitário. IBAMA. Coleção Meio Ambiente. Série Estudos de Pesca.

PETRERE, M. JR. 1992. Pesca na Amazônia. pp. 72-78. Secretaria de Estado de Ciência, Tecnologia e Meio Ambiente - Pará. SIMDAMAZÔNLA, Seminário Internacional Sobre Meio Ambiente, Pobreza e Desenvolvimento da Amazônia. Anais, Belém. PRODEPA. $567 \mathrm{p}$,

ROSS, E.B. 1978. Food taboos, diet, and hunting strategy: the adaptation to animals in Amazon cultural ecology. Current. Antropology 19:1-36.
RUFFINO, M.L. 1992. Relatório de consultoria para o Projeto IARA/ IBAMA. 71 p. (mimeo).

.. 1994. Relatório de consultoria para o Projeto IARA/IBAMA. 58 p. (mimeo).

.....; (no prelo). Desenvolvimento pesqueiro no Baixo Amazonas. In: PADOCH, C.; AYRES, J.M.; HENDERSON, A.; PINEDO-VASQUEZ, M. (eds.). Report of Conference about Diversity, Development and Conservation of the Amazon Floodplain.

SANTOS, G.M.; FERREIRA, E.J.G.; ZUANON, J.A. 1991, Ecologia de peixes da Amazônia. pp.263-280. In: VAL, A.L.; FIGLIUOLO, R.; FELDBERG, E. (eds,). Base cientificas para estratégias de preservação e desenvolvimento da Amazônia: Fatos e perspectivas. INPA, Manaus, $440 \mathrm{p}$.

SHRIMPTON, R.; GIUGLIANO, R. 1979. Consumo de alimentos e alguns nutrientes em Manaus. 1973-4. Acta Amazonica, 9(1):117-141.

SMITH, N.J.H. 1979. Pesca no Rio Amazonas. INPA/CNPq, Manaus. 154 p.

.....; 1981. Man, Fishes and the Amazon. Columbia University Press, New York. $180 \mathrm{p}$.

VIEIRA, I.J.A.; HARTMANN, W.D. 1989. Lago Grande de Monte Alegre: Por uma administração de recursos pesqueiros e, águas interiores da Amazônia. Informe Técnico. IBAMA. Superintendência do Estado do Pará. Setor de Pesca. 17 p.

WELCOMME, R.L. 1985. River fisheries. FAO Fish. Tech. Pap. (262):330 p. 


\section{ANEXO 1}

INSTITUTO BRASILEIRO DO MEIO AMBIENTE E DOS RECURSOS

NATURAIS RENOVÁVEIS -- PROJETO IARA

ESTATISTICA PESQUEIRA - CONSUMO DE ALIMENTOS NAS COMUNIDADES RIBEIRINHAS

Nome da comunidade:

Data:

Nome do Chefe de Família:

№ da casa:

№ de Pessoas Presentes na casa:

Homens (maiores de 12 anos):

Mulheres (maiores de 12 anos):

Cianças (menores de 12 anos):

\begin{tabular}{|c|c|c|c|c|c|}
\hline \multirow{2}{*}{\multicolumn{6}{|c|}{$\begin{array}{l}\text { Tipo de alimento } \\
\text { Acara } \\
\end{array}$}} \\
\hline & & & & & \\
\hline \multicolumn{6}{|l|}{$\begin{array}{l}\text { Acari } \\
\text { Apapa }\end{array}$} \\
\hline \multirow{2}{*}{\multicolumn{6}{|c|}{$\frac{\text { Apapa }}{\text { Aracu }}$}} \\
\hline \multirow{2}{*}{\multicolumn{6}{|c|}{$\frac{\text { Aracu }}{\text { Aruanā }}$}} \\
\hline & & & & & \\
\hline \multicolumn{6}{|l|}{ Branquinha } \\
\hline \multicolumn{6}{|l|}{ Camarāo } \\
\hline \multicolumn{6}{|l|}{ Cara de gato } \\
\hline \multicolumn{6}{|l|}{ Charuto } \\
\hline \multicolumn{6}{|l|}{ Cujuba } \\
\hline \multicolumn{6}{|l|}{ Gurimatã } \\
\hline \multicolumn{6}{|l|}{ Dourado } \\
\hline \multicolumn{6}{|l|}{ Filhote/Piraiba } \\
\hline \multicolumn{6}{|l|}{ Fura Calça } \\
\hline \multicolumn{6}{|l|}{ Jaraqui } \\
\hline \multicolumn{6}{|l|}{ Mapapa } \\
\hline \multirow{2}{*}{\multicolumn{6}{|c|}{$\begin{array}{l}\text { Pacu } \\
\text { Peixe Cachorro }\end{array}$}} \\
\hline & & & & & \\
\hline \multicolumn{6}{|l|}{ Pescada } \\
\hline \multicolumn{6}{|l|}{ Piracatinga } \\
\hline \multicolumn{6}{|l|}{ Piramutaba } \\
\hline \multicolumn{6}{|l|}{ Piranha } \\
\hline \multicolumn{6}{|l|}{ Pirapitinga } \\
\hline \multicolumn{6}{|l|}{ Pirarucu } \\
\hline \multicolumn{6}{|l|}{ Sardinha } \\
\hline Surubim & & & & & \\
\hline Tambaqui & & & & & \\
\hline Tamuata & & & & & \\
\hline Traira & & & 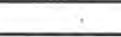 & & \\
\hline Tucunaré & & & & & \\
\hline Outros (Qual)? & & & & & \\
\hline Tracajá & & & & & \\
\hline Ovos de Tracajá & & & & & \\
\hline Jacaré & & & & & \\
\hline Carne de Porco & & & & & \\
\hline Cane de Boi & & & & & \\
\hline Frango & & & & & \\
\hline Piracui & & & & & \\
\hline Farinha & & & & & \\
\hline Tapioca & & & & & \\
\hline Arros & & & & & \\
\hline Batata & & & & & \\
\hline Macarão & & & & & \\
\hline Feijäo & & & & & \\
\hline Ovos & & & & & \\
\hline Leite & & & & & \\
\hline
\end{tabular}


ANEXO 2

Nome comum

acará-açu, acarâ-cascudo, acará-bararuá, acará-bicudo, acará-disco, acará-prata acará-rosado, acará-rouxo, acaratinga
Acarichthys heckelfii, Acaronia nassa, Aequidens sp.,

Astronotus crassipinis, Caquetaia spectabilis,

Chaetobranchus flavescens, Chaetobranchopsis orbicularis,

Cichlasoma amazonarum, Geophagus proximus, Heros $s p$.

Satanoperca acuticeps, S. jurupari, Symphysodon aequifascia,

Uaru amphiacanthoides

Hypostomos emarginatus, Liposarcus pardalis

Pellona flavipinis, $P$. castelnaeana

Leporinus aff. affinis, $L$. fasciatus, $L$, friderici, $L$, trifasciatus,

Rhytiodus argenteofuscus, $R$. microlepis, Schizodon fasctitus, $S$ vittatus

Osteoglossum bizirrosum

aruanã

branquinha comum, cascuda e cabeça lisa

Caenotropus labirinthicus, Curimata inomata, Cyphocharax abramoides, Psectrogaster amazonica, $P$. rutiloides,

Steindachnerina c.f. bimaculata

\begin{tabular}{ll}
\hline camarāo & Machrobrachium spp. \\
\hline cara de gato & Platynematichthys notatus \\
\hline charuto & Anodus melanopogon, Hemiodus immaculatus, H. microlepis, H. \\
& ocellatus, H. unimaculatus, $H . s p$.
\end{tabular}

\begin{tabular}{|c|c|}
\hline cujuba & Oxydoras niger \\
\hline curimatã & Prochilodus nigricans \\
\hline dourado & Brachyplatystoma flavicans \\
\hline filhote e piraiba & Brachyplatystoma whimesntзurr \\
\hline fura calça & Pimelodina flavipinnis, Pimelodus of. altipinnis, P. blocti \\
\hline jaraqui escama fina e escama grossa & Semaprochilodus teanurus, S. insignis \\
\hline jéju & Hoplerythrinus unitaeniatus \\
\hline mapará & Hypophthaimus edentatus, H. fimbriatus, L. marigniatiso \\
\hline pacu comum, jumento e olhudo & $\begin{array}{l}\text { Catoprion mento, Metynnis argenteus, } M \text {. hypsauchen, Myleus } \\
\text { schomburgki. M. torquatus, Mylossoma aureum, } M \text {. duriventre }\end{array}$ \\
\hline peixe cachorro & Raphiodon vulpinus \\
\hline pescada comum e preta & $\begin{array}{l}\text { Pachypops furchraeus, } P \text {. trifilis, Plagioscion auratus, } P \text {, } \\
\text { squamosissimus, } P \text {. surinamensis, } P \text {. sp. }\end{array}$ \\
\hline $\begin{array}{l}\text { piranha branca, caju, mafura, mucura e } \\
\text { preta }\end{array}$ & $\begin{array}{l}\text { Pygocentrus nattereri, Serrasalmus calmoni, S. elongatus, S. } \\
\text { rhombeus. S. spilopleura, S. aff. eigenmmanni }\end{array}$ \\
\hline piracatinga & Calophysus macropterus \\
\hline pirarucu & Arapaima gigas \\
\hline piramutaba & Brachyplathystoma vaillantii \\
\hline pirapitinga & Piaractus brachypomus \\
\hline sardinha comum, comprida e papuda & Triportheus albus, T. elongatus, $T$. flavus \\
\hline $\begin{array}{l}\text { surubim palhaço, surubim flamengo, } \\
\text { surubim lenha ou canela, surubim tigre ou } \\
\text { pintado }\end{array}$ & $\begin{array}{l}\text { Brachyplatystoma juruensi, Merodontotus tigrinus, } \\
\text { Pseudoplatystoma fasciatus, P. tigtrinus, }\end{array}$ \\
\hline tambaqui amarelo ou preto & Colossoma macropomum \\
\hline tamoatá & Hoplosternum littorale \\
\hline traira & Hoplias gr. malabaricus \\
\hline tucunare comum, açu, pinima & Cichla monoculus, C. temensis, C. sp. \\
\hline
\end{tabular}

